\title{
EL DERECHO HUMANO A UN MEDIO AMBIENTE SANO, LA PARTICIPACIÓN PÚBLICA Y EL IUS COMMUNEI
}

\author{
Gonzalo Javier Aguilar Cavallo ${ }^{2}$ \\ Universidad de Talca (UTALCA)
}

\section{RESUMEN}

El derecho humano a disfrutar de un medio ambiente sano, o bien, sin riesgos, limpio, saludable y sostenible se encuentra vinculado con el derecho de acceso a la participación ambiental. La participación pública es un medio para alcanzar el derecho a un medio ambiente sano, pero también forma parte de su contenido. La jurisprudencia de la Corte Interamericana de Derechos Humanos ha desarrollado el derecho a la participación ambiental. De este modo, la Corte ha contribuido a establecer estándares mínimos que forman parte del ius commune de derechos humanos en América Latina.

El objetivo principal de este trabajo es determinar la relevancia y el rol que juega la participación pública en la protección ambiental, así como las normas que lo apuntalan. En este contexto, la pregunta que orientaría el estudio sería ¿cuál es el rol que juega la participación pública en la protección ambiental y cuáles son las fuentes de la misma? El método utilizado será el del análisis dogmático y normativo, junto con la técnica de revisión documental. Encontramos que la satisfacción plena de los derechos de consulta, participación y consentimiento en el ámbito ambiental, son supuestos necesarios para la realización de una democracia real, participativa y deliberativa. Desde el derecho internacional de los derechos humanos comienzan a desarrollarse estándares participatorios ambientales mínimos que se proyectan como comunes para los Estados.

Palabras clave: Corte Interamericana de Derechos Humanos; derecho a la participación ambiental; derecho a un medio ambiente sano; ius commune; participación pública.

1 Este trabajo se enmarca en el Proyecto Fondecyt Regular n. 1190423: Análisis de los estándares internacionales sobre el derecho de acceso a la información, a la participación y a la justicia ambiental y su concreción en el derecho interno, del que el autor es investigador principal.

2 Abogado (Chile), Doctor en Derecho (España), Magister en Relaciones Internacionales (España), Master en Derechos Humanos y Derecho Humanitario (Francia). Post doctorado en el Max Planck Institute for Comparative Public Law and International Law (Heidelberg, Alemania). Profesor de Derecho Constitucional, Internacional, Ambiental y Derechos Humanos, Centro de Estudios Constitucionales de Chile, Universidad de Talca (Santiago, Chile). Director del Magister en Derecho Constitucional del Centro de Estudios Constitucionales de Chile (Santiago, Chile). ORCID: https:// orcid.org/0000-0001-9728-6727 / e-mail: gaguilarch@hotmail.com

Veredas do Direito, Belo Horizonte, $\cdot$ v.16 $\cdot$ n.36 $\cdot$ p.41-66 $\cdot$ Setembro/Dezembro de 2019 
THE HUMAN RIGHT TO A HEALTHY ENVIRONMENT, PUBLIC PARTICIPATION AND IUS COMMUNE

\section{ABSTRACT}

The human right to a healthy environment or the human right to a safe, clean, healthy and sustainable environment is linked to the right to access to environmental participation. Public participation is a mean to exercise the human right to a healthy environment as well as part of its normative content. The Inter-American Court of Human Rights case law has developed environmental participation criteria. Thus, the Court has built up minimum standards which make part of a ius commune on human rights in Latin America. This paper seeks to define the role and relevance of the norms of public participation in the environmental protection. Thus the guiding question is: which role does public participation play in environmental protection and what are the legal sources. Dogmatic analysis is the method used in this paper. The full enjoyment of environmental consultation, participation and consent rights, are requirements for the fulfilment of a real, participative and deliberative democracy. International human rights law has developed participatory minimum standards that are supposed to be shared amongst States.

Keywords: Human Right to a healthy environment; Inter-American Court of Human Rights; ius commune; public participation; right to an environmental participation. 


\section{INTRODUCCIÓN}

El derecho humano a disfrutar de un medio ambiente sin riesgos, limpio, saludable, y sostenible ha venido desarrollándose con fuerza desde la esfera de las Naciones Unidas, especialmente, gracias al trabajo realizado por el Relator Especial de las Naciones Unidas para el medio ambiente y los derechos humanos, John Knox. A partir de los trabajos realizados por el Relator Especial, con el concurso de los Estados miembros de la Organización, el contenido, contornos y límites del derecho humano al medio ambiente se ha ido definiendo y concretizando.

También, desde la esfera internacional, la jurisprudencia internacional ha jugado un rol relevante en los últimos tiempos. Sólo para mencionar un ejemplo, entre varios, la Corte Interamericana de Derechos Humanos (Corte IDH) emitió una Opinión Consultiva sobre el medio ambiente, la cual contiene importantes principios y definiciones en materia de protección del medio ambiente y del derecho a vivir en un medio ambiente sin riesgos, limpio, saludable y sostenible. Sin duda, todas estas definiciones y principios contribuyen a llenar el corpus iuris internacional de los derechos humanos relacionados con el medio ambiente y del derecho humano al medio ambiente en sí mismo considerado. Esta importante evolución que se está produciendo en el ámbito del derecho internacional podría generar un impacto decisivo en el desarrollo futuro del derecho constitucional de los derechos humanos al interior de los Estados. Esto es particularmente relevante, considerando la Opinión Consultiva de la Corte IDH, en los Estados miembros del sistema interamericano de protección de los derechos humanos.

Uno de los rasgos que se ha ido definiendo con mayor precisión en la evolución actual del derecho a un medio ambiente sin riesgos, limpio, saludable, y sostenible ha sido la cada vez más estrecha interdependencia entre los denominados derechos sustantivos ambientales y derechos procesales ambientales, tales como el derecho a la participación ambiental.

El objetivo principal de este trabajo es determinar la relevancia y el rol que juega la participación pública en la protección ambiental, así como las normas que lo apuntalan. En este contexto, la pregunta que orientaría el estudio sería ¿cuál es el rol que juega la participación pública en la protección ambiental y cuáles son las fuentes de la misma? El método utilizado será el del análisis dogmático y normativo, junto con la técnica de revisión documental. 
En este trabajo abordaremos en primer lugar, la conexión entre la protección del medio ambiente y la participación pública, y, posteriormente, la relación entre el derecho humano a un medio ambiente sano y el ius commune.

\section{MEDIO AMBIENTE Y PARTICIPACIÓN PÚBLICA}

En el corazón de la noción de democracia ambiental se encuentra, por cierto, el derecho a la participación, elemento esencial del derecho a la democracia. Y, esta última afirmación se efectúa, no obstante que, en general, la participación ambiental es abordada únicamente desde la perspectiva de los derechos procedimentales en el contexto ambiental (WAITE, 2008, p. 57). E incluso, a veces, se realiza la distinción entre participación política y participación administrativa, intentando separar ambas (JORDANO, 1995, p. 192). Quizás, una alternativa a esta visión sea el enfoque de derechos, en el sentido de que todos los derechos humanos son interdependientes e interrelacionados. Por tanto, el derecho a la información, el derecho a la participación y el derecho al acceso a la justicia ambiental son todos derechos humanos cuyo respeto protección y satisfacción es necesaria para la realización plena del derecho a un medio ambiente sano. Incluso más, Sarlet y Almagro (2013, p. 383) afirman que "los derechos fundamentales son proyección material en el Estado constitucional del principio democrático".

Se podrían esgrimir distintos títulos legítimos que justificarían la participación ambiental. Así, desde la perspectiva española y considerando la legitimidad del interés ambiental (el individuo como titular de intereses propios - uti singulus - o titular de intereses como miembro de la comunidad - uti cives) se ha afirmado que

[...] cuando un ciudadano participa en un procedimiento (en sentido amplio), esgrimiendo su derecho a un medio ambiente adecuado, es portador al mismo tiempo de una posición jurídica individual (el derecho al medio ambiente consagrado por el artículo $45 \mathrm{CE}$, como derecho público subjetivo) y de una posición jurídica colectiva. $\mathrm{Y}$ es que un derecho o interés colectivo tiene a la vez por titular al individuo y a la comunidad. Esta matización no es simplemente terminológica, pues la importante consecuencia que de ella se deriva consiste en el fortalecimiento de la posición desde la que se participa y el propio contenido de la participación. Esta no sólo será la posibilidad de esgrimir datos desconocidos para la Administración, o de ser oído a los efectos de conformar la decisión administrativa soberana, burocrática y discrecional, sino también, y esto es lo más importante, la posibilidad de controlarla 
desde parámetros sustantivos de sujeción no meramente reaccionales (JORDANO, 1995, p. 196).

La participación de los individuos de una comunidad, entendida como el ejercicio permanente de un derecho a un gobierno democrático, es consubstancial a la democracia, es lo que la explica, es lo que la mantiene y es su fin continuado (FRANCK, 1992, p. 46; JACKSON, 2002, p. 304; Vid. FOX y ROTH, 2000). Se acerca un poco a esta noción global del derecho a la participación lo señalado por Costa y Fuentes (2011, p. 86), cuando estos entienden "por participación ciudadana aquellas actividades voluntarias mediante las cuales los miembros de la sociedad civil, pueden intervenir fundamentalmente en tres esferas: en la elección de sus gobernantes (directa o indirectamente), en la gestión pública y en el ejercicio del control ciudadano". Este aspecto del control es fundamental para explicar la relevancia de la participación pública ambiental (que algunos la consideran como participación funcional), ya que "el alcance, calidad y contenido de las relaciones entre la sociedad y su entorno dependerán en buena medida de la potenciación y del ejercicio de los medios que la participación funcional ofrece como mecanismo de control y de influencia en la aplicación del Derecho" (JORDANO 1995, p. 200). Sarlet y Almagro (2013, p. 386), por su parte, llenan de contenido la participación política del ciudadano siguiendo una triple vertiente:

a) de forma directa (principalmente mediante las figuras jurídicas del referendo, el plebiscito y la iniciativa legislativa popular);

b) de forma indirecta, mediante la elección de los representantes que conformarán la voluntad de los órganos representativos del Estado;

c) mediante la directa incorporación del ciudadano a los poderes estatales o bien a la función pública.

La participación pública tiene la ventaja de dotar al proceso de toma de decisiones de transparencia, equidad y legitimidad, permitiendo el fortalecimiento de vínculos al interior de la comunidad, precisamente porque encuentra su justificación en el ejercicio mismo del derecho a la participación democrática, del gobierno por propio consentimiento y del principio de soberanía popular. Esto último adquiere una dimensión particular, por el interés creciente de la protección y preservación ambiental al interior de la comunidad. Así, Costa y Fuentes destacan que una de las ventajas de la participación pública en el proceso de toma de decisiones en asuntos ambientales es que permite 
Incorporar al proceso de evaluación los conocimientos, propuestas de la comunidad local; mejorando los proyectos. Por lo demás, se promueven relaciones, diálogo, intercambio y cooperación entre la comunidad, el proponente y los servicios públicos; se fortalecen las capacidades de la ciudadanía; se amplía y canaliza la entrega de información de acuerdo a las características propias de cada comunidad, y; se reviste al proceso de toma de decisiones de transparencia y legitimidad, estableciendo relaciones armónicas con la comunidad (COSTA y FUENTES, 2011, p. 83- 106, p. 89; MIROSEVIC, 2011, p. 285).

\subsection{Concepto}

La participación ambiental es una especie cada vez más relevante de ejercicio específico del derecho humano a la participación en los asuntos ambientales, los cuales constituyen una función pública, por definición constitucional e internacional. Detrás de este derecho se encuentra la noción del gobierno por consentimiento del pueblo.

La participación ambiental encuentra indudablemente sus fuentes jurídicas en los pilares más profundos tanto del derecho constitucional como internacional.

\subsection{Las fuentes}

El derecho a la participación pública es un principio cardinal en un Estado constitucional y democrático de derecho. En el Estado constitucional del siglo XXI opera el principio del primado del derecho y de los derechos humanos. El derecho a la participación pública, especialmente en el ámbito medioambiental, encuentra su reconocimiento tanto en el derecho internacional como constitucional.

\subsubsection{Fuentes internacionales}

La Declaración de la Conferencia de las Naciones Unidas sobre el Medio Humano realizada en Estocolmo, en 1972, reconoce en su Principio 1 que

[e]l hombre tiene derecho fundamental a la libertad, la igualdad y el disfrute de condiciones de vida adecuadas en un medio de calidad tal que le permita llevar una vida digna y gozar de bienestar, y tiene la solemne obligación de proteger y mejorar el medio para las generaciones presentes y futuras. 


\section{Esta misma Declaración de Estocolmo señala en su Recomendación} 97, que el Secretario General de las Naciones Unidas debería

[e]stablecer un programa de información destinado a suscitar el interés de los particulares por el medio humano y a lograr la participación del público en su ordenación y control. Tal programa recurrirá a los medios de información pública tradicionales y contemporáneos, teniendo en cuenta las peculiaridades nacionales. Además, deberá prever los medios de estimular la participación activa de los ciudadanos y despertar el interés y lograr la contribución de las organizaciones no gubernamentales en la salvaguardia y el mejoramiento del medio.

Además, el principio elemental de participación ambiental encuentra sus fundamentos de derecho internacional, inter alia, en la Carta Mundial de la Naturaleza de 1982. En efecto, el Principio 23 de este instrumento establece que

[t]oda persona, de conformidad con la legislación nacional, tendrá la oportunidad de participar, individual o colectivamente, en el proceso de preparación de las decisiones que conciernan directamente a su medio ambiente y, cuando éste haya sido objeto de daño o deterioro, podrá ejercer los recursos necesarios para obtener una indemnización ${ }^{3}$.

\section{Del mismo modo, el Principio 10 de la Declaración de Río de 1992 señala que}

[e]1 mejor modo de tratar las cuestiones ambientales es con la participación de todos los ciudadanos interesados, en el nivel que corresponda. En el plano nacional, toda persona deberá tener acceso adecuado a la información sobre el medio ambiente de que dispongan las autoridades públicas, incluida la información sobre los materiales y las actividades que encierran peligro en sus comunidades, así como la oportunidad de participar en los procesos de adopción de decisiones. Los Estados deberán facilitar y fomentar la sensibilización y la participación de la población poniendo la información a disposición de todos. Deberá proporcionarse acceso efectivo a los procedimientos judiciales y administrativos, entre éstos el resarcimiento de daños y los recursos pertinentes.

Desde el punto de vista europeo, el Principio 10 antes mencionado se ha incorporado a la Convención sobre acceso a la información, participación pública en la adopción de decisiones y acceso a la justicia en asuntos ambientales, adoptado en Aarhus, en 1998 (CONVENCIÓN DE AARHUS). ${ }^{4}$ Este Convenio tiene por objetivo contribuir a proteger el derecho de cada persona, de las generaciones presentes y futuras, a

3 La Carta Mundial de la Naturaleza fue aprobada por la Asamblea General de las Naciones Unidas mediante Resolución 37/7 de 28 de octubre de 1982.

4 La Convención fue adoptada en la Conferencia Ministerial "Medio Ambiente para Europa" celebrada en Aarhus, Dinamarca, el 25 de junio de 1998. 
vivir en un medio ambiente que permita garantizar su salud y su bienestar, garantizando los derechos de acceso a la información sobre el medio ambiente, la participación del público en la toma de decisiones y el acceso a la justicia en asuntos ambientales (CONVENCIÓN DE AARHUS, art. $\left.1^{\circ}\right)$

\subsubsection{Fuentes constitucionales}

El articulo $1^{\circ}$ inciso $4^{\circ}$ de la Constitución Política de la República de Chile señala que es deber del Estado "[...] promover la integración armónica de todos los sectores de la Nación y asegurar el derecho de las personas a participar con igualdad de oportunidades en la vida nacional".

Esta misma consagración constitucional del derecho a la participación se verifica en el derecho comparado. Por ejemplo, desde este último punto de vista, en México, Brañes (2000, p. 165) ha sostenido que "la participación social en la gestión ambiental encuentra sus fundamentos jurídicos en diversos artículos constitucionales, como el 4, 25, 9, 40 y 41 ". Vera, Lina y Conraud (2009, p. 193) han agregado que "estos últimos dos prevén que el pueblo ejerce su soberanía a través de los Poderes de la Unión, en los casos de la competencia de éstos, y por los Estados, en lo relativo a sus regímenes interiores, en los términos establecidos por la propia Constitución y las de los Estados".

\subsection{Participación social}

La participación social es fundamental en los procesos vinculados a la toma de decisiones en relación con asuntos ambientales. La participación social implica el involucramiento directo de la comunidad afectada en el proceso de toma de decisiones y en la adopción final de la decisión misma. Tal como Bishop (1981, p. 88) señalara hace más de 30 años, “como regla general se debería proporcionar la oportunidad de participar a todos. El público podría elegir participar o no participar. Es su prerrogativa. Pero el Estado (sic) debería asegurar que esta elección fuera siempre posible para el público" (BISHOP, 1981, p. 87). Ahora bien, Echavarren (2007, p. 110) ha sostenido que "[u]na forma de obtener información y cooperación por parte de la comunidad local es a través de la participación social, un apartado que es inherente a la evaluación de impacto ambiental". Pero la participación social que se propone en la toma de decisiones ambientales 
va más allá de una mera difusión o comunicación social o bien, de una capacidad de búsqueda y entrega de información ambiental. Bishop, por ejemplo, tiende a identificar participación con comunicación social. Así, este autor ha afirmado que "desde el momento en que la participación pública es esencialmente un proceso de comunicación social, sin la identificación del público involucrado en este proceso, no puede operar efectivamente" (BISHOP, 1981, p. 87). ${ }^{5}$ En esta línea, Echavarren nos menciona cuatro posibles modelos de comunicación con el grupo o comunidad afectada por el impacto ambiental del proyecto.

Se observan cuatro modelos de procesos de comunicación: 1) modelo de difusión, donde se concentran las fases de provisión de información; 2) modelo de recogida, donde se incluyen los procesos de retroalimentación, identificación y evaluación de problemas entre otros; 3) modelo de interacción, que engloba los mecanismos de logro de consenso social; y 4) modelo de difusión-recogida, donde se hacen públicas las conclusiones del informe de evaluación (ECHAVARREN, 2007, p. 110).

Por cierto que resulta muy relevante identificar la comunidad afectada, que puede ser local, regional, estatal, o incluso internacional. Pero, a nuestro entender la participación social implica, en último término, ya sea por consenso, aceptación o asentimiento, un control sobre la decisión ambiental.

La participación de la comunidad en el proceso de toma de decisiones no es sino el reflejo del grado de democratización de una sociedad. Por su parte, Bishop (1981, p. 93) ha planteado que la participación social cumple cinco objetivos:

1. promover la información, educación y coordinación;

2. identificar posibles problemas, necesidades y valores;

3. posibilitar el flujo de nuevas ideas y resolución de problemas;

4. posibilitar una participación más democrática de las comunidades afectadas y la evaluación de alternativas del proyecto de desarrollo que les afecta;

5. ayudar a la creación de un consenso social y la resolución de conflictos (ECHAVARREN, 2007, p. 110).

Cuando la decisión se refiere a asuntos ambientales la participación se puede precisar como ambiental y la democracia al interior de dicha comunidad - cualquiera que sea su nivel organizacional - también como ambiental.

La participación social, entonces, es un requisito sine qua non de la toma de decisiones ambientales, sin cuya presencia la decisión carece de

5 "This advice is of prime importance. Since public participation is essentially a social communication process, without the identification of the publics involved in this process it cannot operate effectively". 
validez. Así, Echavarren ha sostenido que

[1]a participación social no es entonces un factor socioeconómico de la Evaluación de Impacto Ambiental, sino un requisito de la misma, que indica la profundidad del análisis del impacto social llevado a cabo, y una muestra de la democratización de la sociedad que la ha llevado a cabo. La participación social es una constante de la Evaluación de Impacto Ambiental en la legislación de todos los países donde se aplica, con mayor o menor importancia. La mínima expresión de la participación pública en las Evaluaciones de Impacto Ambiental, y que es práctica común en muchas de ellas, es la de proporcionar información del desarrollo del proyecto en cuestión con anterioridad a su implementación y la de admitir alegaciones al respecto (ECHAVARREN 2007, p. 110).

En esta línea, Irigalba, Etxaleku y Echavarren (2002, p. 361) han manifestado que "la participación pública cumple varios objetivos. Uno de los más importantes es el de la información". Y, vinculado con lo anterior, han agregado que "el mismo hecho de que la comunidad tome conciencia de que tiene algún poder sobre las decisiones públicas, tiene su importancia social". En efecto, la participación social es un requisito que dota de legitimidad al proceso de toma de decisiones y a la decisión misma, y que estrecha, por tanto, los lazos de confianza entre los diferentes actores de una sociedad. Así, la participación es un factor esencial de legitimidad del ejercicio del poder y la participación ambiental lo es respecto de la toma de decisiones ambientales. La legitimidad social de la decisión ambiental acarreará su amplia aceptación social. Entendida de esta manera la participación social en asuntos ambientales, ésta sólo puede redundar en un fortalecimiento de la cohesión social.

\subsubsection{La importancia de tomar en cuenta la valoración social del proyecto}

Hemos observado que la participación social permite tomar en consideración de manera fundamental la voluntad de la comunidad afectada por el impacto ambiental de un proyecto. Esta participación tiene la virtud de ser un medio concreto de ejercer la democracia y reviste tanto el proceso como la decisión final de un aurea de legitimidad, no sólo jurídica sino sobre todo democrática. Esta legitimidad constituye una de las razones que justifican tomar en cuenta la valoración social del proyecto. Desde este punto de vista, Echavarren propone que una evaluación de impacto ambiental de los aspectos socioeconómicos debería incluir, al menos, el estudio y análisis de los siguientes factores: en primer lugar, la calidad de 
vida de la población lo que podría incluir aspectos tales como los estilos de vida, la identidad social, el turismo, la salud, los valores sociales, etc. En este aspecto, examinando fundamentalmente el artículo 45 de la Constitución Española, Jordano (1995, p. 106) ha distinguido claramente dos conceptos que se encuentran íntimamente vinculados, a saber, calidad de vida y medio ambiente, indicando que el medio ambiente, su protección, restauración y mejora se comprende como un elemento indispensable del concepto de calidad de vida, pero no el único. En segundo lugar, la demografía. En tercer lugar, la economía y la población activa, lo que podría considerar aspectos tales como infraestructuras o usos del suelo. En cuarto lugar, los factores culturales, lo que podría incluir aspectos tales como sitios arqueológicos, sitios históricos, elementos arquitectónicos, patrimonios naturales, etc. En quinto lugar, el paisaje; y, en sexto lugar, las relaciones de la comunidad social con su medioambiente biofísico (ECHAVARREN, 2007, p. 103). Echavarren agrega que la consideración de cada uno de estos factores y el peso que se le asigna en cada estudio variará según cada situación, porque la evaluación de impacto ambiental es un instrumento que debe caracterizarse por su flexibilidad y que debe, por tanto, adaptarse lo mejor posible a cada caso en particular (ECHAVARREN, 2007, p. 103). Es así como Pardo (1994, p. 164) ha sostenido que

[s]ería igualmente importante el cambio en el enfoque rígido de la participación pública, cuya base fundamental es la exposición pública del documento en los locales del Ayuntamiento y similares, a un enfoque mucho más abierto en donde, a través de formas participativas más dinámicas e interactivas, se incorpore la valoración social de proyectos y su negociación.

Desde el momento en que la participación pública es una expresión de la democracia y una manifestación concreta del ejercicio de la soberanía, en cuanto poder exclusivo y excluyente del pueblo, la participación pública, como se viene de señalar, en los procesos ambientales, debería ser flexible, dinámica y abierta de tal manera de admitir las distintas posibilidades de inclusión de la participación de todos los involucrados. Esto último se encontraría en sintonía con la propuesta de Häberle (2010, p. 381) de una democracia ciudadana. Desde este punto de vista, esta visión de la participación pública coincide con la noción de una sociedad democrática dentro de un constitucionalismo abierto. ${ }^{6}$ Del mismo modo, esta participación se inserta en un constitucionalismo dinámico, sujeto a la

6 "Habermas affords civil society an important role in opinion-formation within the public sphere, mediating between the 'lifeworld' and the political 'system"” (ARMSTRONG, 2006, p. 47). 
diversidad cultural de la sociedad (WARLEIGH, 2006, p. 68; MÜLLER y SCHEPPELE, 2008, p. 67; HABERMAS, 1996; 2001, p. 5).

\subsubsection{El diálogo, la negociación y la aceptación social del proyecto}

La participación pública en los procesos decisionales relativos a asuntos ambientales no sólo tiene una justificación democrática, como se ha observado anteriormente, sino también netamente jurídica, en el sentido de respetar el derecho a la autodeterminación, consagrado en el articulo $1^{\mathrm{o}}$ tanto del Pacto Internacional de Derechos Civiles y Políticos como del Pacto Internacional de Derechos Económicos, Sociales y Culturales. En este sentido, la participación pública se convierte en la medida de una sociedad democrática en el siglo XXI, de tal manera que mientras mayor, en cantidad y calidad, es la participación de individuos, comunidades y pueblos, mejor es la democracia de esa sociedad. Así las cosas, la participación pública se transforma también en la fuente de la armonía y del consenso social, en el sentido de que la participación, directa o indirectamente en la toma de decisiones, genera la consecuente aceptación social de los efectos o impactos que producen las decisiones ambientales adoptadas.

Vinculado con la participación social en los asuntos ambientales, Dron se refiere a la elección de los riesgos aceptables o al estado aceptable del medio ambiente, y hace hincapié en que esta elección, en una sociedad democrática, debe ser tomada con la mayor concertación posible. En esta línea de la aceptación social de la decisión (riesgosa) medioambiental, Dron, citando a Sagoff, recurre a la figura de decir que 'hay una diferencia moral entre saltar y ser empujado.' (DRON, 2000, p. 109; SAGOFF, 1988). La mayor convocatoria posible implica una transformación de la forma tradicional de ejercicio del poder y de adopción de decisiones. Los conocimientos, saberes y opiniones, admitidos e incluso imprescindibles, para la toma de decisiones ambientales implican la consideración de otros interlocutores distintos de aquellos que tradicionalmente acceden a los decidores. Lo anterior significa cuestionar y desafiar los procesos clásicos de toma de decisiones (DRON, 2000, p. 110).

En este mismo contexto de alcanzar el consenso necesario o bien, la mayor concertación en el ámbito medioambiental, que implica generar la mayor convocatoria posible, Dron (2000, p. 110) alude a la negociación social en materia ambiental y señala que esta no es simétrica y que levanta complejidades procedimentales específicas (Vid. OST, 1995). 
Pardo (1994, p. 164) también sostiene que la evaluación de impacto ambiental debería sobre todo considerar "la aceptación social del proyecto (sic) para que se tenga el input de la participación pública desde las fases más incipientes del proyecto". La participación pública permite o facilita, entre otras cosas, la aceptación comunitaria de los impactos ambientales. Así, González (2006, p. 124) ha sostenido que la participación tiene "el propósito de lograr la aceptación social de los impactos generados por el nuevo desarrollo; la comprensión, cambio de opinión y transformación de actitudes y prácticas".

De esta manera la participación pública en los procesos de propuesta, planificación, análisis y decisión de proyectos con impactos en el medio ambiente no sólo aumentan la legitimidad democrática de la decisión ambiental sino también tendrían por virtualidad evitar el conflicto socioambiental. Un proceso ambiental abierto a la participación más amplia posible, transparente y respetuoso de la voluntad de la comunidad afectada generaría, sin duda, confianza en los gobernados. En este sentido, Echavarren (2007, p. 111) ha sostenido que

[e]n el proceso de participación social, es esencial la confianza y credibilidad del equipo investigador, que se conseguirá proporcionando a todos los grupos y subgrupos oportunidades similares de colaborar en el proceso y de la misma manera, es necesario que la posterior redacción de conclusiones tenga una forma (o apariencia) lo más objetiva posible.

De esta manera, además, se cumpliría con el principio constitucional de confianza y de satisfacción de las expectativas legítimas (BERGE y WIDDERSHOVEN, 1998, p 422; BEVIER, 1980, p. 482; QUINOT, 2004, p. 65). A este respecto se ha sostenido que "uno de los principios fundamentales de la Constitución es que un Estado se rija por el Estado de derecho (Rechtsstaat). El principio primordial de un Estado gobernado por el principio del Estado de derecho (Rechtsstaat) es la protección de los derechos del pueblo, el mantenimiento del orden jurídico y la adherencia a los principios de honestidad y buena voluntad. La legítima confianza del pueblo en los resultados del ejercicio del poder por las autoridades debe ser adecuadamente protegido por el derecho; éste es el rationale del principio de las expectativas legítimas (Vertrauenschutzprinzip)" (CONSTITUTIONAL COURT OF TAIWAN, 2001, Case n. 525). 


\subsection{La participación ambiental, complejizar el sistema y el proceso para democratizarlo}

Existen diversos niveles de participación, configurándose así el derecho a la participación en la conducción de los asuntos públicos, como un abanico de derechos o bien en un derecho multifacético y multidimensional. En efecto, la participación pública puede adquirir distintas dimensiones que van desde un simple acceso a la información, hasta la adopción misma de la decisión por la comunidad afectada. Con todo, cabe hacer presente que desde un punto de vista material y desde la perspectiva del ejercicio democrático de la soberanía, es el pueblo o comunidad afectada quien adopta, en último término, la decisión final sobre sus destinos.

Sin embargo, desde un punto de vista procedimental, la implementación concreta del derecho a la participación puede adquirir distintos grados o sujetarse a una ejecución gradual. En este contexto, aparecen como formas concretas de participación pública, el derecho a la consulta y el derecho al consentimiento libre, previo e informado. ¿Cuál es la relación entre participación, consulta y consentimiento? La relación entre ellos es simplemente de intensidad o grados de participación, pero son todos aspectos o dimensiones específicas del derecho humano a la participación, el cual, a su vez, es una manifestación del derecho a la autodeterminación. Esto que se predica respecto de la participación democrática en general, resulta totalmente aplicable a la participación ambiental.

Incluso, desde el punto de vista de la participación en las decisiones ambientales, es donde se ha planteado con mayor fuerza que grados poco intensos de participación, tales como la consulta, no son suficientes. Se ha propuesto que en el ámbito ambiental se requiere, con especial urgencia y necesidad - planteada así por la propia comunidad -, avanzar hacia grados más intensos de participación, donde no sólo se pida la opinión de la comunidad o pueblo, sino que - para dotar de seriedad al ejercicio democrático de este derecho - se deba obtener el consentimiento de los afectados. Por esta razón, se ha sostenido que "[n]o parece serio que se denomine participación pública a lo que se viene haciendo" (PARDO, 1994, p. 164).

De este modo, se ha afirmado también que evidentemente la consulta pública en los procesos de evaluación de impacto ambiental es una especie del género de la participación social (VERA, LINA MANJARREZ y CONRAUD, 2009, p. 193). Y, a su vez, podría afirmarse que la 
participación social es una especie del género de la participación pública. Así, Costa y Fuentes (2011, p. 88) sugieren que existen distintos niveles de participación ambiental, según el grado de influencia de los actores en la decisión ambiental, a saber, el nivel informativo o unidireccional, el nivel consultivo o bidireccional y, por último, el nivel resolutivo o vinculante. En efecto,

[e]n primer lugar, se encuentra el nivel informativo en el cual una de las partes, el proponente o autoridades, entrega información sobre el proyecto y sus impactos. Ejemplo de esto son, los boletines, trípticos y anuncios radiales. En este nivel, la información se entrega de forma unidireccional sin posibilidades inmediatas de solucionar dudas o precisar otros aspectos. En segundo lugar, encontramos el nivel consultivo de Participación Ciudadana como aquel en virtud del cual los interesados forman parte de un proceso durante el cual podrán entregar recomendaciones, formular observaciones y despejar dudas, sin ser obligatorio en la toma de decisiones. En este nivel se establece una relación informativa bidireccional entre los actores. Finalmente el nivel resolutivo, el cual implica que las partes involucradas tengan el mismo grado de incidencia en la toma de decisiones. En este nivel, los participantes entregan una opinión de carácter vinculante que debe ser ejecutada por la autoridad.

Por su parte, Pardo (1994) nos propone tres niveles de participación social en las diferentes fases de la evaluación ambiental de proyectos dentro del proceso de evaluación de impacto ambiental. En primer lugar, plantea la participación en el nivel de la viabilidad; en segundo lugar, plantea la participación en el nivel de estudio de alternativas de localización o a la totalidad del proyecto; $y$, por último, propone el nivel de desarrollo del proyecto (PARDO, 1994). En cada uno de estos tres niveles de participación Pardo (1994) sugiere tres fases o etapas de participación, a saber, consultas previas, estudio de aceptación social y, por último, participación pública como tal. Nosotros nos atrevemos a sugerir una cuarta etapa que se intercalaría luego de las consultas previas o preliminares, y antes de la aceptación social, referido a un estudio de no vulneración de los derechos humanos, tanto de individuos como de grupos o comunidades, lo que ciertamente aumentaría las probabilidades de superar la siguiente etapa relativa a la aceptación social. En definitiva, a partir de Pardo, proponemos una participación en cuatro etapas en el proceso -ahora complejo- de evaluación de impacto ambiental: En primer lugar, consultas previas; en segundo lugar, estudio de no vulneración de derechos humanos; en tercer lugar, estudio de aceptación social; y, en cuarto lugar, participación pública como tal. Tal como señala Pardo (1994, p. 164) "[u]n enfoque así complejiza el proceso, por supuesto, pero desde 
luego lo democratiza y, en última instancia, lo hace más eficaz en cuanto a la posibilidad real de detección de impactos, como ya se ha indicado con anterioridad, y en cuanto a la legitimación social de los proyectos de desarrollo". Por esta misma razón, es que Echavarren (2007, p. 105) señala que la "definición del ámbito de estudio de los factores socioeconómicos es una tarea compleja".

\section{DERECHO HUMANO A UN MEDIO AMBIENTE SANO Y IUS COMMUNE}

En esta parte abordaremos el examen de las normas de derechos humanos como el nuevo derecho común, especialmente, singularizado en el derecho interamericano y en la naturaleza constitucional del rol de protección jurisdiccional de los derechos humanos, de modo de justificar que todo órgano jurisdiccional que protege los derechos humanos desempeña una función constitucional.

\subsection{Derechos humanos y derecho común}

Los derechos humanos se encontrarían a la base de un nuevo ius commune. En este sentido Serna de la Garza (2013.p.36) frente a la pregunta ¿Qué es el ius commune?, ha planteado la siguiente respuesta:

En primer lugar, podemos mencionar que los países del sistema interamericano de derechos humanos comparten en sus Constituciones una serie de valores comunes, centrados en la dignidad de la persona humana y los derechos que de ella irradian. En segundo lugar, existe un bloque normativo común a los Estados adscritos al sistema interamericano de protección de los derechos humanos: la Convención Americana de Derechos Humanos y la jurisprudencia de la Corte Interamericana de Derechos Humanos (SERNA DE LA GARZA, 2013, p. 36).

El gran jurista Jorge Carpizo definió claramente esta noción de ius constitutionale commune latinoamericanum, señalando lo siguiente:

La Convención Americana y su interpretación de última instancia, realizada por la Corte Interamericana de Derechos humanos (Corte IDH), está creando un ius commune latinoamericano de derechos humanos, que implica que cualquier habitante de la región goza de un mínimo de derechos que la Constitución de cada país puede ampliar, pero nunca restringir, en virtud de las obligaciones internacionales que cada Estado contrajo al ratificar la propia Convención Americana y aceptar la jurisdicción obligatoria de la Corte IDH (BOGDANDY, 2013, p. 43). 
Este ius commune se construiría sobre la base de la noción de estándares mínimos comunes y compartidos, por los Estados partes del sistema interamericano de protección de los derechos humanos. Estos estándares comunes y compartidos vendrían determinados por el derecho positivo, por ejemplo, por la Convención Americana sobre Derechos Humanos de 1969, y por la interpretación que de ella se hace por el órgano autorizado para ello (COMISIÓN INTERAMERICANA DE DERECHOS HUMANOS, 2011, p. 2). El órgano autorizado para la interpretación de la Convención Americana sobre Derechos Humanos es la Corte Interamericana de Derechos Humanos.

¿Por qué los derechos humanos constituyen un terreno fértil para el desarrollo de un ius commune en América Latina? Existirían, al menos, cuatro elementos que permitirían, eventualmente, el surgimiento de un ius commune en derechos humanos.

1. Una lengua común. La herencia de la colonización ibérica en América nos legó ya sea el español o bien el portugués. Esta comunidad lingüística, inexistente en Europa, facilita enormemente no sólo las comunicaciones, sino que sobre todo permite la existencia de una comunidad de pensamientos, de ideas, incluso una unión en torno a la expresión lingüística de valores.

2. Una base cultural común. En términos generales, América latina tiene el mismo origen étnico, una parte indígena y una parte resultado de olas de colonización, fundamentalmente de la península ibérica. América Latina es el resultado de un mismo proceso de colonización iniciado desde la época de Cristóbal Colón. Este hecho ha generado una marca indeleble en la región de la que dan cuenta sus poblaciones actuales. Fundamental, en este proceso de desarrollo cultural latinoamericano ha sido la religión católica la que ha convivido con restos aun presentes de creencias indígenas. A pesar de que los pueblos indígenas han bordeado la extinción en América, su capacidad de resistencia les ha permitido sobrevivir, fundamentalmente mezclados con las poblaciones de inmigrantes y esclavos, y en partes, sin mezclarse con otras poblaciones. Este último es un dato clave en América Latina, donde la cosmovisión indígena corre por la sangre de la región, y, por lo tanto, la visión de comunidad se encentra fuertemente arraigada, del mismo modo que en África.

3. Una idiosincrasia común. Las características distintivas y propias de la colectividad americana justifica la presencia de una visión común frente 
a la posición que juega el individuo y la comunidad. En este punto, la situación de América Latina es similar a la de África, donde el aspecto comunitario ocupa un lugar relevante en las relaciones humanas.

4. El elevado índice de desigualdad e inequidad existente en las sociedades latinoamericanas. En efecto,

América Latina sigue conformada por países en desarrollo, con grandes sectores sociales ubicados por debajo de la línea de pobreza, altos niveles de concentración del ingreso y con el nivel de desigualdad más pronunciado del mundo. Sin embargo es notorio que, a pesar de las dificultades existentes, por primera vez una región en desarrollo está -en su totalidad- organizada políticamente de forma democrática. Las democracias pobres de la región obligan a pensar la economía y la democracia en términos propios, para no caer en la equivocación de creer que la democracia tiene más resistencia de la que en realidad cuenta, o que las transformaciones económicas pueden hacerse independientemente de lo que siente y expresa una sociedad determinada (DDI, 2016, p. 519).

$\mathrm{Si}$ aceptamos que comunidad implica la existencia de valores comunes y compartidos y que estos valores son los que le dan su identidad y la cohesión social a esta comunidad, entonces podríamos sostener que en América Latina existiría una comunidad de derechos humanos, en la medida que reconozcamos que los derechos humanos representan los valores centrales de una comunidad, a la luz de los cuales ésta es modelada. En esta línea, se ha sostenido que en los países de la región, en mayor o menor grado, las dinámicas modernizadoras y la secularización del Estado desplazan hacia otros ámbitos los sentidos de pertenencia y la comunidad de valores.

Uno de estos ámbitos que hoy determina la agenda y el debate, dado el avance de la democracia y del Estado de derecho, es el de la ciudadanía moderna. Indudablemente esto supone avanzar en la plena universalidad de los derechos civiles, políticos, económicos, sociales y culturales, lo que requiere conjugar el Estado de derecho, el respeto a las libertades, la representación política y el mayor acceso a oportunidades de bienestar, de uso productivo de capacidades y de protección social. La titularidad de los derechos sociales encarna la efectiva pertenencia a la sociedad, pues implica que todos los ciudadanos estén incluidos en la dinámica del desarrollo y gocen del bienestar que este desarrollo promueve (OTTONE, 2007, p. 23).

Estos valores comunes y compartidos se ven expresados en los principales instrumentos internacionales de derechos humanos adoptados en la región. En el nombre de sus pueblos, los Estados representados en la IX Conferencia Internacional Americana, señalan en el preámbulo de la Carta de la Organización de los Estados Americanos (OEA) de 1948, que 
están seguros que "el sentido genuino de la solidaridad americana y de la buena vecindad no puede ser otro que el de consolidar en este Continente, dentro del marco de las instituciones democráticas, un régimen de libertad individual y de justicia social, fundado en el respeto de los derechos esenciales del hombre".

Al momento de aprobar la Declaración Americana de los Derechos y Deberes del Hombre (DADDH) de 1948, la IX Conferencia Internacional Americana consideró que "los pueblos americanos han dignificado la persona humana y que sus constituciones nacionales reconocen que las instituciones jurídicas y políticas, rectoras de la vida en sociedad, tienen como fin principal la protección de los derechos esenciales del hombre y la creación de circunstancias que le permitan progresar espiritual y materialmente y alcanzar la felicidad", y que "la protección internacional de los derechos del hombre debe ser guía principalísima del derecho americano en evolución". Cabe resaltar la formula contenida al inicio del preámbulo de la Declaración, consistente en que "[t]odos los hombres nacen libres e iguales en dignidad y derechos y, dotados como están por naturaleza de razón y conciencia, deben conducirse fraternalmente los unos con los otros". Como se sabe, una retórica similar es contenida en el artículo $1^{\circ}$ de la Constitución chilena.

Por su parte, los Estados signatarios de la Convención Americana sobre Derechos Humanos de 1969 reafirman su "propósito de consolidar en este Continente, dentro del cuadro de las instituciones democráticas, un régimen de libertad personal y de justicia social, fundado en el respeto de los derechos esenciales del hombre".

La Carta Democrática Interamericana de 2001 señala en su preámbulo que "la solidaridad y la cooperación de los Estados americanos requieren la organización política de los mismos sobre la base del ejercicio efectivo de la democracia representativa y que el crecimiento económico y el desarrollo social basados en la justicia y la equidad y la democracia son interdependientes y se refuerzan mutuamente", y reafirma que "la lucha contra la pobreza, especialmente la eliminación de la pobreza crítica, es esencial para la promoción y consolidación de la democracia y constituye una responsabilidad común y compartida de los Estados americanos".

Y, recientemente, la Declaración Americana sobre los Derechos de los Pueblos Indígenas de 2016, donde los Estados miembros de la OEA reafirman que "los pueblos indígenas son sociedades originarias, diversas y con identidad propia que forman parte integral de las Américas". 


\subsection{Derecho a un medio ambiente sano}

La Corte IDH ha contribuido decisivamente a la construcción de un corpus iuris interamericanum en el ámbito del derecho humano a un medio ambiente sano con su Opinión Consultiva sobre medio ambiente y derechos humanos n. 23/17, de fecha 15 de noviembre de 2017.

En efecto, en esta Opinión Consultiva, la Corte IDH (2017, pars. 62 y 63 ) ha afirmado, en el nivel internacional, el derecho a un medio ambiente sano como un derecho humano autónomo:

Esta Corte considera importante resaltar que el derecho al medio ambiente sano como derecho autónomo, a diferencia de otros derechos, protege los componentes del medio ambiente, tales como bosques, ríos, mares y otros, como intereses jurídicos en sí mismos, aún en ausencia de certeza o evidencia sobre el riesgo a las personas individuales. Se trata de proteger la naturaleza y el medio ambiente no solamente por su conexidad con una utilidad para el ser humano o por los efectos que su degradación podría causar en otros derechos de las personas, como la salud, la vida o la integridad personal, sino por su importancia para los demás organismos vivos con quienes se comparte el planeta, también merecedores de protección en sí mismos. En este sentido, la Corte advierte una tendencia a reconocer personería jurídica y, por ende, derechos a la naturaleza no solo en sentencias judiciales sino incluso en ordenamientos constitucionales. De esta manera, el derecho a un medio ambiente sano como derecho autónomo es distinto al contenido ambiental que surge de la protección de otros derechos, tales como el derecho a la vida o el derecho a la integridad personal.

Asimismo, la Corte IDH (2017, par. 59) ha reiterado que el derecho humano a un medio ambiente sano presenta aspectos tanto individuales como colectivos:

El derecho humano a un medio ambiente sano se ha entendido como un derecho con connotaciones tanto individuales como colectivas. En su dimensión colectiva, el derecho a un medio ambiente sano constituye un interés universal, que se debe tanto a las generaciones presentes y futuras. Ahora bien, el derecho al medio ambiente sano también tiene una dimensión individual, en la medida en que su vulneración puede tener repercusiones directas o indirectas sobre las personas debido a su conexidad con otros derechos, tales como el derecho a la salud, la integridad personal o la vida, entre otros. La degradación del medio ambiente puede causar daños irreparables en los seres humanos, por lo cual un medio ambiente sano es un derecho fundamental para la existencia de la humanidad.

Por último, cabe destacar que los jueces interamericanos han resaltado que el derecho a un medio ambiente sano acarrear determinadas y precisas 
obligaciones para los Estados (aunque también alude a las obligaciones de las empresas). En efecto,

[...] el derecho al medio ambiente sano, tal como está previsto en el Protocolo de San Salvador (sic), conlleva las siguientes cinco obligaciones para los Estados: a) garantizar a toda persona, sin discriminación alguna, un medio ambiente sano para vivir; b) garantizar a toda persona, sin discriminación alguna, servicios públicos básicos; c) promover la protección del medio ambiente; d) promover la preservación del medio ambiente, y e) promover el mejoramiento del medio ambiente. Asimismo, ha establecido que el ejercicio del derecho al medio ambiente sano debe guiarse por los criterios de disponibilidad, accesibilidad, sostenibilidad, aceptabilidad y adaptabilidad, común a otros derechos económicos, sociales y culturales. A efectos de analizar los informes de los Estados bajo el Protocolo de San Salvador, en 2014 la Asamblea General de la OEA aprobó ciertos indicadores de progreso para evaluar el estado del medio ambiente en función de: a) las condiciones atmosféricas; b) la calidad y suficiencia de las fuentes hídricas; c) la calidad del aire; d) la calidad del suelo; e) la biodiversidad; f) la producción de residuos contaminantes y manejo de estos; g) los recursos energéticos, y h) el estado de los recursos forestales" (CORTE IDH, 2017, par. 60).

\section{CONCLUSIONES}

Principios elementales que se encuentran a labase del constitucionalismo contemporáneo, tales como la soberanía popular y el gobierno por propio consentimiento o bien, el principio de la democracia real y el derecho a la participación, el cual se encuentra en la médula de este principio, constituyen el sustratum de la democracia ambiental y concretamente del derecho a la participación efectiva en la preparación, planificación, diseño, aprobación y ejecución de los proyectos o propuestas de desarrollo o inversión, particularmente, a través de los sistemas de evaluación de impacto ambiental. En definitiva, la satisfacción plena de los derechos de consulta, participación y consentimiento en el ámbito ambiental, específicamente a través de los procesos de evaluación ambiental, no sólo importan el respeto de los derechos humanos vinculados a la protección del medio ambiente sino más aun, son supuestos necesarios para la realización de una democracia real, participativa y deliberativa.

Estos mismos principios se proyectan para justificar el derecho a la consulta, participación y consentimiento de los pueblos indígenas en los casos de afectación mediante proyectos de inversión o desarrollo, especialmente proyectos extractivos, sobre sus tierras, territorios y recursos 
naturales. La plena satisfacción de estos derechos humanos se erige como una vía adecuada para evitar o hacer frente a conflictos ambientales y, de esa menara, concretar la gobernanza ambiental. La gobernanza ambiental tiene como supuesto la garantía del cumplimiento del principio de carácter constitucional de las legítimas expectativas de la población, que no refleja sino la confianza que tienen los individuos de que el Estado, sin discriminación, protegerá y garantizará los derechos de todos.

En nuestra visión, los derechos humanos constituyen el nuevo derecho común del siglo XXI. Vemos un principio de orden constitucional allí donde existen, al menos como principio, derechos humanos. Por ello, debido a que, desde el punto de vista de la dignidad humana, resulta difícil pensar en normas más propiamente constitucionales que los derechos humanos, es que hemos sostenido que estos derechos serían enunciados normativos con carácter propiamente constitucional. Esto destaca la importancia de traer los derechos humanos a casa, en el entendido que estos derechos representan estándares mínimos exigidos por la dignidad humana.

En el derecho constitucional contemporáneo, uno de los rasgos identificadores de la jurisdicción constitucional es la protección de los derechos humanos fundamentales. En este sentido, los jueces que efectúan un rol concreto en la protección de los derechos humanos desempeñan una función constitucional. Si aceptamos, como lo hace la Corte IDH, que existe un derecho humano al medio ambiente sano, como derecho autónomo, con fuente y fundamento en los instrumentos interamericanos de derechos humanos, entonces, este derecho ha pasado a formar parte del ius commune latinoamericanum. En este sentido, los parámetros y definiciones que nos aporten los jueces interamericanos a propósito de este derecho serán fundamentales, al interior de cada uno de los Estados partes del sistema interamericano de protección de los derechos humanos, para configurar el grado de protección mínima de este derecho humano al medio ambiente sano. Resta por ver si esta visión material del derecho, centrada en la protección no sólo formal sino también sustantiva de los mismos, y, por lo tanto, su rol de protección de primer orden, es asumida por los jueces nacionales, quienes se convertirían, en este caso, en jueces comunes interamericanos de derechos humanos. 


\section{REFERENCIAS}

ARMSTRONG, K. A. Inclusive governance? Civil society and the open method of co-ordination. In: SMISMANS, S. (Ed.). Civil society and legitimate European governance. Cheltenham: Edward Elgar, 2006. p. 42$-67$.

BRAÑES BALLESTEROS, R. Manual de derecho ambiental mexicano. México: Fundación Mexicana para el Derecho Ambiental-Fondo de Cultura Económica, 2000.

BERGE, G.; WIDDERSHOVEN, R. The Principle of Legitimate Expectations in Dutch Constitutional and Administrative Law. Netherlands Reports to the Fifteenth International Congress of Comparative Law, $\mathrm{p}$. 422-452, 1998.

BISHOP, A. B. Communication in the planning process. In: CREIGHTON, J. L.; DELLI PRISCOLI, J. D. (Eds.). Public involvement techniques: a reader of ten years experience at the Institute of Water Resources. Fort Belvoir: U.S. Army Engineer Institute for Water Resources, 1981. p. 81-98 .

BEVIER, L. R. An Informed Public, an Informing Press: The Search for a Constitutional Principle. 68 Cal. L. Rev. 482, 1980, p. 482-517

BOGDANDY, A. Ius constitutionale comune latinoamericanum. Una aclaración conceptual desde una perspectiva europea. In: GONZÁLEZ PÉREZ, L. R.; VALADÉS, D. (Coords.). El constitucionalismo contemporáneo. Homenaje a Jorge Carpizo. México: UNAM-IIJ, 2013. p. 39-66.

COMISIÓN INTERAMERICANA DE DERECHOS HUMANOS. Estándares jurídicos vinculados a la igualdad de género y a los derechos de las mujeres en el Sistema Interamericano de Derechos Humanos: desarrollo y aplicación. Doc. OEA/Ser.L/V/II.143 Doc. 60, 2011.

CONSTITUTIONAL COURT OF TAIWAN. Constitutional Interpretation. Case n. 525, 4 Mai 2001.

CORTE INTERAMERICANA DE DERECHOS HUMANOS. Medio ambiente y derechos humanos (obligaciones estatales en relación con el medio ambiente en el marco de la protección y garantía de los derechos a la vida y a la integridad personal - interpretación y alcance de los 
artículos 4.1 y 5.1, en relación con los artículos 1.1 y 2 de la Convención Americana sobre Derechos Humanos). Opinión Consultiva OC-23/17 de 15 de noviembre de 2017. Serie A No. 23.

COSTA CORDELLA, E.; FUENTES MERINO, P. La participación ciudadana en las declaraciones de impacto ambiental. Revista de Derecho Ambiental, año III, n. 3, sep., p. 83-106, 2011.

DECLARACIÓN DE RIO DE JANEIRO SOBRE EL MEDIO AMBIENTE Y EL DESARROLLO. Conferencia de las Naciones Unidas sobre el Medio Ambiente y el Desarrollo, Rio de Janeiro del 3 al 14 de junio de 1992.

DEPARTAMENTO DE DERECHO INTERNACIONAL DE LA SECRETARÍA DE ASUNTOS JURÍDICOS DE LA OEA (DDI). Las dimensiones de la democracia, 2016 p. 519. Disponible en: $<$ https://www. oas.org/dil/esp/afrodescendientes_Manual_Formacion_Lideres_anexos. pdf $>$. Accedido el 21 septiembre 2016.

DRON, D. Environnement: les enjeux du prochain siècle. Ramses 2001, septembre 2000, p. 95-114.

ECHAVARREN, J. M. Aspectos socioeconómicos de la Evaluación de Impacto Ambiental. Revista Internacional de Sociología (RIS), v. LXV, n. 47, p. 99-116, mayo-ago. 2007.

FOX, G. H.; ROTH BRAD, R. (Eds.). Democratic governance and International Law. Cambridge, U.K.: Cambridge University Press, 2000.

FRANCK, T. M. The emerging right to democratic governance. American Journal of International Law, v. 86, n. 1, p. 46-91, 1992.

GONZÁLEZ HERRERA, M. La evaluación como impacto ambiental como instrumentos de gestión de destinos turísticos. Teoría y Praxis, n. 2, p. 105-126, 2006.

HÄBERLE, P. Métodos y principios de interpretación constitucional: un catálogo de problemas. Revista de Derecho Constitucional Europeo, año 7, n. 13, p. 379-411, enero-junio 2010.

HABERMAS, J. Between facts and norms: contributions to a discourse theory of law and democracy. Cambridge: Polity Press, 1996.

HABERMAS, J. Why Europe Needs a Constitution. New Left Review, v. 11, p. 5-26, 2011. 
INFORME DE LA CONFERENCIA DE LAS NACIONES UNIDAS SOBRE EL MEDIO HUMANO. Estocolmo, 5 a 16 de junio de 1972.

IRIGALBA,A.C.; ETXALEKU,A. I.; ECHAVARREN, J. M. La evaluación de impacto ambiental: recopilación, análisis y punto de vista crítico desde la perspectiva sociológica. In: ALEDO TUR, A.; DOMÍNGUEZ GÓMEZ, J. A. (Eds.). Sociología ambiental. Madrid: Grupo Editorial Universitario, 2002. p. 361-403.

JACKSON, W. D. Democratic governance and International Law (review). Human Rights Quarterly, v. 24, n. 1, p. 304-312, 2002.

JORDANO FRAGA, J. La protección del derecho a un medio ambiente adecuado. Barcelona: Bosch, 1995.

MIROSEVIC, C. La Participación Ciudadana en el Procedimiento de Evaluación de Impacto Ambiental y las Reformas introducidas por la Ley n. 20.417. Revista de Derecho de la Pontificia Universidad Católica de Valparaíso, v. XXXVI, p. 281-323, 2011.

MÜLLER, J.-W.; SCHEPPELE, K. L. Constitutional patriotism: an introduction. International Journal of Constitutional Law, n. 1, p. 67-71, 2008 .

OST, F. La nature hors-la-loi: l'écologie à l'épreuve du droit. Paris: La Découverte, 1995.

OTTONE, E. (Dir.). Cohesión social: inclusión y sentido de pertenencia en América Latina y el Caribe. Santiago: CEPAL, Naciones Unidas, 2007.

PARDO BUENDÍA, M. El impacto social en las Evaluaciones de Impacto Ambiental: su conceptualización y práctica. Reis: Revista Española de Investigaciones Sociológicas, n. 66, p. 141-167, 1994.

QUINOT, G. Substantive legitimate expectations in South African and European Administrative Law. German Law Journal, n. 5, n. 1, p. 65-85, 2004.

SAGOFF, M. The Economy of the Earth. Cambridge: Cambridge University Press, 1988.

SARLET, I. W.; ALMAGRO CASTRO, D. Los derechos políticos en España y Brasil: una aproximación en perspectiva comparada. Estudios Constitucionales, año 11, n. 1, p. 381-424, 2013. 
SERNA DE LA GARZA, J. M. Jorge Carpizo y el proyecto Hacia un Ius Commune Latinoamericano en Derechos Humanos. In: GONZÁLEZ PÉREZ, L. R.; VALADÉS, D. (Coords.). El constitucionalismo contemporáneo. Homenaje a Jorge Carpizo. México: UNAM-IIJ, 2013. p. 35-38.

VERA MORALES, L. R.; LINA MANJARREZ, P.; CONRAUD, T. Propuesta de mejora al proceso de la participación ciudadana dentro de la evaluación del impacto ambiental. Revista Legislativa de Estudios Sociales y de Opinión Pública, v. 2, n. 4, p. 187-203, dic. 2009.

WAITE, A. Sunlight through the trees: a perspective on environmental rights and human rights. DAIBERT, A. (Org.). Direito Ambiental comparado. Belo Horizonte: Forum, 2008. p. 47-66.

WARLEIGH, A. Civil society and legitimate governance in a flexible Europe: critical deliberativism as a way forward. In: SMISMANS, S. (Ed.). Civil society and legitimate European governance. Cheltenham: Edward Elgar, 2006. p. 68-86.

Artículo recibido en: 15/07/2019.

Artículo aceptado en: 12/08/2019.

\section{Cómo citar este artículo (ABNT):}

CAVALLO, G. J. A. El derecho humano a un medio ambiente sano, la participación pública y el ius commune. Veredas do Direito, Belo Horizonte, v. 16, n. 36, p. 41-66, set./dez. 2019. Disponible en: http://www.domhelder. edu.br/revista/index.php/veredas/article/view/1598. Acceso en: día de mes de año. 